\title{
Radiation tests on the HAS2 image sensor with colour filter array for the JUICE Monitoring Cameras
}

Pawel Adamiec, Nikhil Banerji, Juan Barbero, Stephane Beauvivre, Enrique Cordero, et al.

Pawel Adamiec, Nikhil Banerji, Juan Barbero, Stephane Beauvivre, Enrique Cordero, Monica Rodriguez Cortina, Morten Andreas Geday, Daniel Krähenbühl, Alejandro Lopez Moya, Ruth Ziethe, "Radiation tests on the HAS2 image sensor with colour filter array for the JUICE Monitoring Cameras," Proc. SPIE 11852, International Conference on Space Optics ICSO 2020, 118525E (11 June 2021); doi: 10.1117/12.2599949

SPIE Event: International Conference on Space Optics - ICSO 2021, 2021, Online Only 


\section{International Conference on Space Optics-ICSO 2020}

Virtual Conference

30 March-2 April 2021

Edited by Bruno Cugny, Zoran Sodnik, and Nikos Karafolas
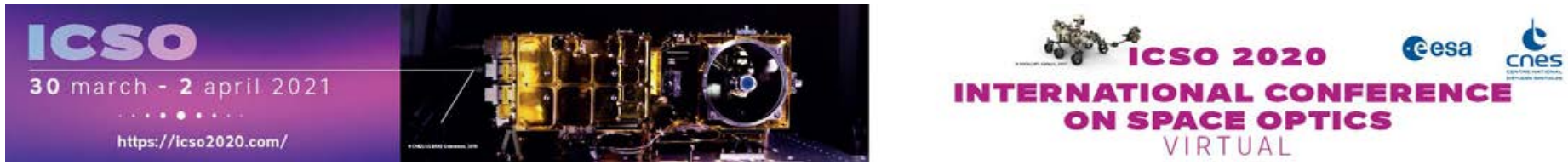

\section{Radiation tests on the HAS2 image sensor with colour filter array for the JUICE Monitoring Cameras}

\section{Cesa isoporecestings denes}




\title{
Radiation tests on the HAS2 image sensor with color filter array for the JUICE Monitoring Cameras
}

Pawel Adamiec*a, Nikhil Banerjic ${ }^{\mathrm{c}}$, Juan Barbero ${ }^{\mathrm{a}}$, Stephane Beauvivre ${ }^{\mathrm{c}}$, Enrique Cordero ${ }^{\mathrm{a}}$, Monica Rodriguez Cortina ${ }^{\mathrm{a}, \mathrm{b}}$,Morten Andreas Geday ${ }^{\mathrm{b}}$, Daniel Krähenbühl ${ }^{\mathrm{c}}$, Alejandro Lopez Moya ${ }^{\mathrm{a}}$, and Ruth Ziethe ${ }^{\mathrm{c}}$

\author{
aALTER TECHNOLOGY TÜV NORD S.A.U., C/ la Majada 3, 28760 Tres Cantos, Spain; \\ ${ }^{\mathrm{b}}$ CEMDATIC, ETSI Telecomunicación, Universidad Politécnica de Madrid, Av. Complutense 30, \\ 28040 Madrid, Spain; \\ ${ }^{\mathrm{c}}$ Micro-Cameras and Space Exploration SA, Puits-Godet 10a, CH-2000 Neuchâtel, Switzerland
}

\begin{abstract}
A radiation hard image sensor HAS2 was chosen for the Juice Monitoring Camera, an important instrument to monitor the status of the JUpiter ICy moons Explorer (JUICE) spacecraft and to take wide-angle images of the Jovian system. To improve the perception, a radiation-hardened, non-organic color filter array (CFA) was deposited on the sensor silicon, with four colors: red, green, blue, and yellow. In this paper we present the results of the radiation tests on these customized image sensors. First, results on the total ionizing dose (TID) test up to $57 \mathrm{krad}$ confirm that both the image sensor and the deposited CFA are resistant to gamma radiation in the measured range. Also, single event effects (SEE) measurements up to LET of $100 \mathrm{MeV} \cdot \mathrm{cm}^{2} / \mathrm{mg}$ were performed. Three types of events were considered: single event latch-up (SEL), single event upset (SEU), and finally single event functional interruption (SEFI). Since only one SEL was registered on one of the samples at $62.5 \mathrm{MeV} \cdot \mathrm{cm}^{2} / \mathrm{mg}$ there was no further analysis on this event. Based on the experimental results, the cross sections have been calculated for SEU and SEFI. These results were used as input for the SEE analysis with the CRÈME96 software. The rates of about $10^{-4}$ and $10^{-5}$ events/device/day were obtained for SEU and SEFI for quiet environment, respectively.
\end{abstract}

Keywords: Radiation hard, image sensor, HAS2, JUICE, total ionizing doze, single event effect

\section{INTRODUCTION}

The JUpiter ICy moons Explorer (JUICE) is an ESA interplanetary spacecraft under development. Its mission is to study the Jovian system. Besides the scientific instruments, onboard equipment has been foreseen to monitor and assess the status and health of the spacecraft. The Juice Monitoring Cameras will be used to monitor the deployment of the solar panels and antennae, as well as to acquire wide-angle color images of the Jovian system. Due to the high radiation levels in the Jovian system in comparison to the earth orbits (LEO and GEO) [1], a radiation hard image sensor is essential for this mission. Hence, a CMOS image sensor (CIS) was chosen which has an inherently higher resistance to the radiation exposure than a charge-coupled device (CCD) due to single charge transfer in comparison to multi-charge transfer in CCDs. There were already CIS used in some missions targeting: (i) the Earth orbit like COBRA image sensor used in GOCI instrument in COMS mission [2] and VNIR instrument in Sentinel2 mission [3], (ii) inner reaches of our solar system like SRI CIS used in SoloHI and WISPR instruments in Solar Orbiter [4], (iii) and recently SIRIUS (CIS115) was chosen for JANUS instrument in the JUICE mission [5]. Since mentioned imagers were custom developments with special efforts in time and costs, Micro-Cameras and Space Exploration SA identified and focused its JUICE development on existing image sensors with space heritage to address its client need. The HAS2 sensor has flown on the PROBA-2 mission [6] and was successfully tested under different irradiation conditions like gamma radiation, protons, electrons, neutrons, and heavy ions (HI) [7-10]. All these tests confirmed that this sensor is well suited for space missions with elevated radiation environments like the Jovian system.

*pawel.adamiec@altertechnology.com 
To meet not only the pure monitoring requirement but also meet the objective of color images, even for science purposes, the commercially available HAS2 sensor was upgraded by deposition of radiation-hardened color filters. From the initial calculation, it was estimated that during the JUICE mission the sensor will suffer maximum TID of 55 krad [11]. Section 2 describes the samples and the experimental setups including the elegant breadboard camera. The TID results are presented in the Section 3, while the SEE results in the Section 4. Finally, the review of the results and performances is presented in conclusions.

\section{SAMPLE AND SETUPS DESCRIPTION}

\subsection{Sample description}

HAS2 is a radiation-tolerant CMOS monochrome image sensor with 1024x1024 pixels array. The pixel is a photodiodetype with the size of $18 \mu \mathrm{m} \times 18 \mu \mathrm{m}$ and 3T readout circuit. It supports rolling shutter operation with 12-bit ADC and has been described in the following references [7-10]. For the JUICE application it was decided to deposit a color filter array (CFA) on top of the photodiode array. To increase the information content of images four colors were chosen: red, green, blue, and yellow. The filters were deposited on the top of the wafers, which were then cut into single image sensors and assembled in a custom 84-pin ceramic package (see Figure 1).

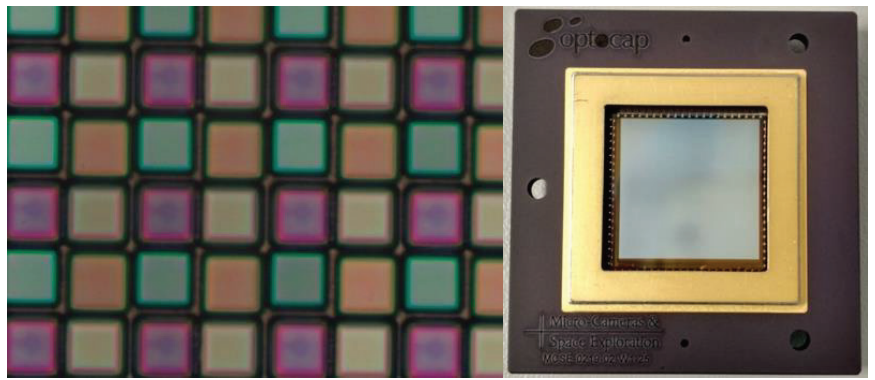

Figure 1. Color filer array (left) and assembled image sensor inside the ceramic package (right).

The image sensor has four $3.3 \mathrm{~V}$ power rails, namely: VDD, VPIX, VRES (reset), and VAA.

\subsection{Camera elegant breadboard}

The test camera set-up is an elegant breadboard developed by ALTER TECHNOLOGY and consists of necessary electronics to communicate with the image sensor, e.g. FPGA, memory, UDP, and USB to access the registers via SPI protocol. The sensor is placed in a remote socket, with necessary passive elements for decoupling (see Figure 2), making the breadboard flexible for different testing set-ups such as radiation, thermal vacuum, or thermal cycling analysis. The image sensor was powered using a high precision power supply from Keithley, 2230G-30-1, monitoring currents and voltages by means of a Data Acquisition Unit Keysight 34970A.

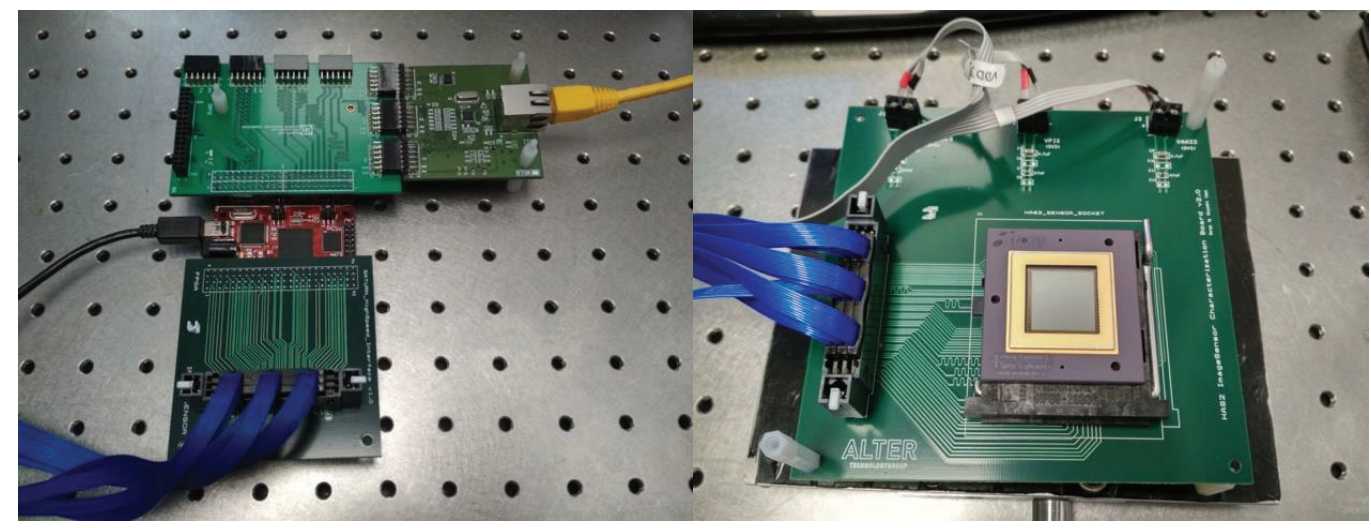

Figure 2. Camera elegant breadboard (left) and remote board with the image sensor (right). 


\subsection{Optical setup and optical measurements}

The optical setup for image sensor characterization was developed at ALTER according to the EMVA Standard 1288 Standard for Characterization of Image Sensor and Cameras, Release 3.0, November 29, 2010. One of the provisions of the EMVA standard is that each pixel color is characterized separately. Therefore, the setup consisted of a light source with four colors (LEDs) integrating sphere (Newport 819-SF-6), power meter (Newport 2936-C) with photodetector (Newport 883-SL), and spectrometer (Thorlabs CCS200). The linearity of the light source is better than 0.995 measured from the center of the image sensor to the edges. The setup and its realization are shown in the Figure 3 . The incident light properties were measured by means of the power meter and the spectrometer and reads: red $-630 \mathrm{~nm}-1.466 \mu \mathrm{W}$, yellow $-590 \mathrm{~nm}-1.688 \mu \mathrm{W}$, green $-535 \mathrm{~nm}-2.454 \mu \mathrm{W}$, and blue $-465 \mathrm{~nm}-4.561 \mu \mathrm{W}$. The full width at half maximum $(\mathrm{FWHM})<50 \mathrm{~nm}$ for each color. The exposure times used in the experiments for photon transfer method (PTM) were set up to $14.5 \mathrm{~ms}$ where the saturation of the flat images occurred at all colors. For the dark current (DC) measurements longer exposure times were used up to $120 \mathrm{~ms}$. For spatial nonuniformities, namely photo response nonuniformity (PRNU) and dark signal nonuniformity (DSNU) the images were taken at $50 \%$ of the saturation capacity.

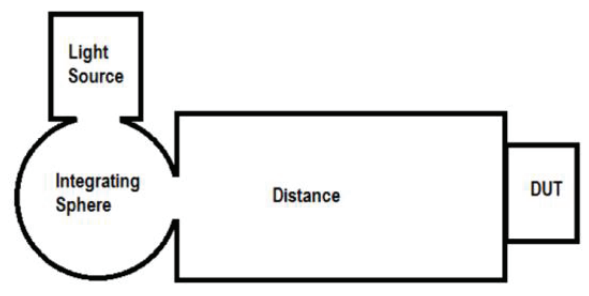

(a) Setup Sechematic

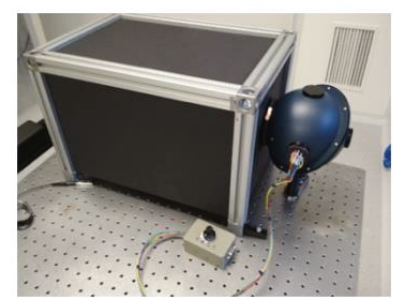

(b) Setup photo 1

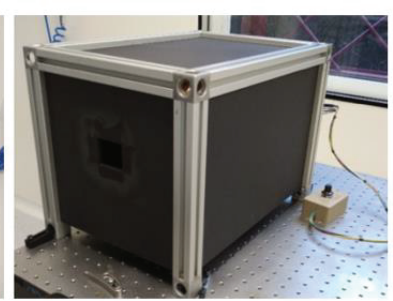

(c) Setup photo 2

Figure 3. Schematic and pictures of the setup for optical measurements.

\subsection{Radiation setups}

The TID test was performed at CIEMAT's radiation facility in Madrid, Spain. It is the NAYADE installation of the water pool type and ${ }^{60} \mathrm{Co}$ sources placed at the bottom of the pool. The samples were placed inside hermetic vessels. The internal cavity protects the samples and permits the uniformity of the dose rates. Four samples: 2 biased and 2 unbiased were fixed to the metallic plate inside the vessel and the connections were taken outside by hermetically sealed wire tubes (about $10 \mathrm{~m}$ in length). The setup is shown in the Figure 4. The dose rate was about $235 \mathrm{rad} / \mathrm{h}$ resulting in a 5 krad daily dose, considering 2 hours break for the imagers characterization. The intermediate characterization was taken at $10 \mathrm{krad}, 20 \mathrm{krad}$, $36 \mathrm{krad}$, and the final one after $57 \mathrm{krad}$. After the 48-h annealing at room temperature (RT), one week (168 h) annealing was performed at $85^{\circ} \mathrm{C}$.

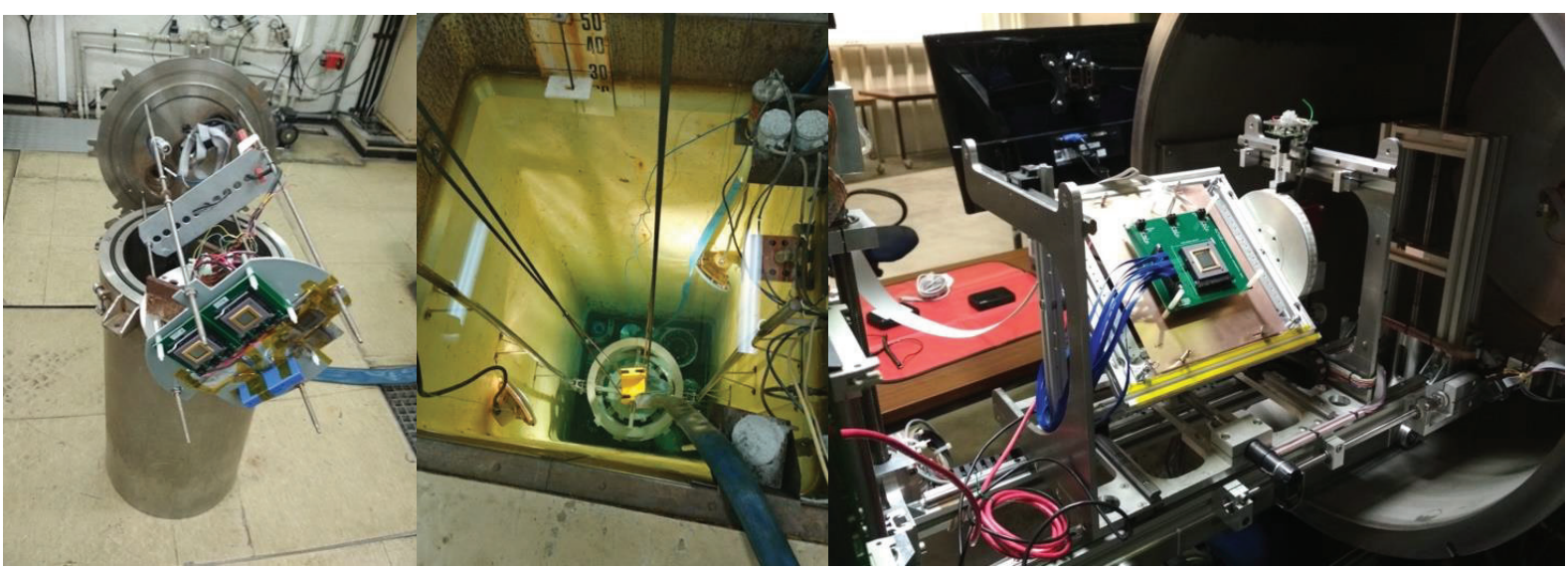

Figure 4. TID setup: (left) samples on the holder and hermetic vessel, (middle) the vessel inside the pool; SEE setup (right) camera with the image sensor mounted on a holder inside the vacuum chamber. 
According to the requirements of the JUICE mission the Single Event Effects (SEE) should be tested in the LET range from 1 to $100 \mathrm{MeV} \cdot \mathrm{cm}^{2} / \mathrm{mg}$. The maximum LET for heavy ions (HI) available in Europe (at UCL, Belgium) is $62.5 \mathrm{MeV} \cdot \mathrm{cm}^{2} / \mathrm{mg}$. Therefore, the tilting of the sample was necessary. The beam had about $2.5 \mathrm{~cm}$ of diameter and was entering the vacuum chamber where the DUT was placed. Inside the vacuum chamber were placed the PCB with the image sensor and its motherboard. The sensor was connected using 3 supply lines, VDD, VAA, and VPIX (which was connected to the VRES). The motherboard was accessed through Ethernet and a modem, the later controlled by a PC for image transfer. The motherboard was also connected via USB to the PC providing power and SPI communication. The power supply and monitoring block was used to drive the image sensor and monitor the currents and voltages during the SEE tests in order to detect the SEL. The picture of the setup is shown in the Figure 4. The board with DUT was fixed inside the vacuum chamber to the moving stage, which allows for positioning inside the beam and rotation to the desired angle to increase the LET. The flux of the HI beam can be set from tens to $10,000 \mathrm{ions} /\left(\mathrm{cm}^{2} \cdot \mathrm{s}\right)$.

\section{TOTAL IONIZING DOSE}

The TID test was performed on 4 samples. Two samples \#1 and \#2 were irradiated under bias, whereas other two: \#3 and \#4 short circuited. During the irradiation test, only the power rails were connected and monitored, the clocks were not routed, and the images were not acquired. During the intermediate measurements, at every step the electrical measurements were taken, a flat image for each filter color to check the responsivity and 16 images at every exposure time for dark current calculation were acquired. The exposure times were set to $1 \mathrm{~ms}, 20 \mathrm{~ms}, 40 \mathrm{~ms}, 60 \mathrm{~ms}, 80 \mathrm{~ms}, 100 \mathrm{~ms}$, and $120 \mathrm{~ms}$. The same measurements were repeated after each annealing. The evolution of the dark current for all imagers is shown in the Figure 5. The units of dark current are given in $\mathrm{DN} / \mathrm{s}(\mathrm{DN}$ - dark numbers, for 12-bit images taking values from 0 black pixel to 4095-white pixel).

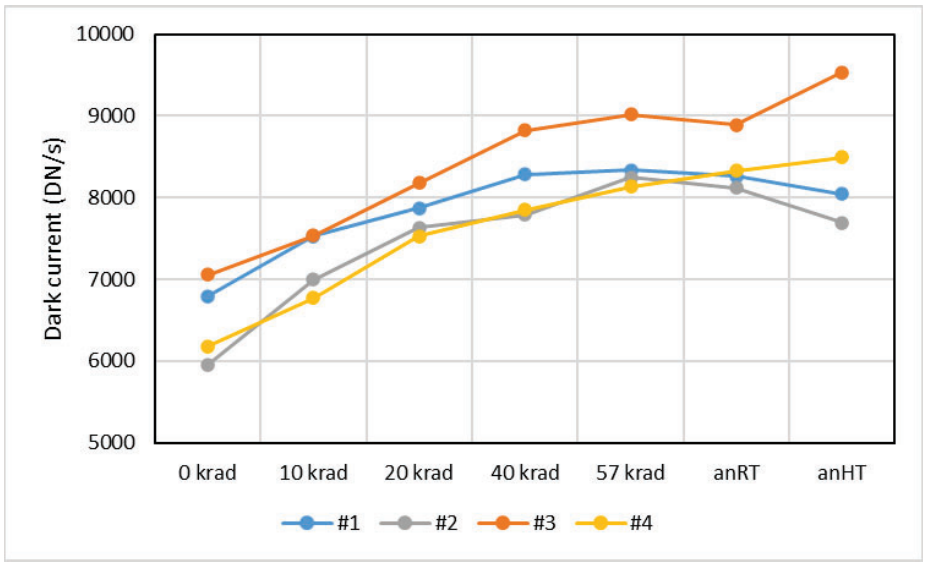

Figure 5. Dark current evolution during the TID test. First five correspond to the radiation steps and last two to the annealing steps: anRT - annealing at room temperature and anHT - annealing at $85^{\circ} \mathrm{C}$.

The measurements were taken at different room temperatures $t$, therefore the dark current was corrected using actual temperature and the doubling temperature $t_{\mathrm{d}}=9.8^{\circ} \mathrm{C}$ with the following equation

$$
D C_{\text {corr }}(t)=D C(t)+D C(t) \cdot\left[\left(t_{0}-t\right) / t_{\mathrm{d}}\right],
$$

where $t_{0}$ is the temperature of the initial measurement. As expected, the DC was increasing until the last radiation step. The relative change was of $23 \%$ and $39 \%$ for biased samples $\# 1$ and $\# 2$, whereas it read $28 \%$ and $32 \%$ for unbiased image sensors \#3 and \#4, respectively. After annealing, the imagers which were biased during the TID test recovered partially to the relative values of $18 \%$ and $29 \%$ for sample \#3 and \#4, respectively. Surprisingly, the unbiased imagers showed further increase of DC up to $36 \%$ of the initial value. The imagers were fully characterized by means of PTM at the beginning and after all the annealing tests. The thorough analysis of the results of the biased and unbiased samples did not indicate any significant difference, therefore we will present only average results per color pixel. Table 1 shows the comparison between initial and final results for each color. After filter deposition the most indicative parameter of the CFA quality is the responsivity and thus the quantum efficiency. The red pixels show the highest initial responsivity of $0.2 \mathrm{~W} / \mathrm{A}$, whereas the blue ones show 3 times lower which reads $0.06 \mathrm{~W} / \mathrm{A}$. This difference is also reflected in the quantum efficiency where red 
pixels reached about $40 \%$ and the blue ones $16 \%$. After TID test the responsivity remains unchanged for all the pixels confirming the radiation hardness of the CFA. The most affected parameter is the saturation capacity which drops about $10 \%$ after the TID test, resulting in slight decrease in the dynamic range (2\%) and $\mathrm{SNR}_{\max }$ (about $1 \%$ ). The linearity error decreased slightly after the TID test, but the change is so small and rather compared to the computing error than real improvement of the image sensor. Concerning the spatial uniformities, the DSNU did not change after the radiation test and read 4.2 DN, while the PRNU before and after the test read: $0.56 \%$ and $0.57 \%$ for whole image sensor which was well below $1 \%$ necessary for the mission and indicated that the imagers should sustain the foreseen radiation levels during the JUICE mission.

Table 1. Comparison of PTM parameters after TID test.

\begin{tabular}{|l|l|l|l|l|l|l|c|}
\hline Color & Test & Responsivity & $\begin{array}{c}\text { Quantum } \\
\text { efficiency }\end{array}$ & $\begin{array}{c}\text { Saturation } \\
\text { capacity }\end{array}$ & $\begin{array}{c}\text { Dynamic } \\
\text { range }\end{array}$ & $\begin{array}{c}\text { Linearity } \\
\text { error }\end{array}$ & $\begin{array}{c}\text { SNR } \\
\text { max }\end{array}$ \\
\hline \multirow{3}{*}{ Yellow } & & \multicolumn{1}{|c}{$(\mathrm{A} / \mathrm{W})$} & & $(\mathrm{e}-)$ & $(\mathrm{dB})$ & $(\%)$ & $(\mathrm{dB})$ \\
\cline { 2 - 8 } & final & 0.15 & 0.31 & 67913 & 58.1 & 1.5 & 48.3 \\
\hline \multirow{2}{*}{ Green } & initial & 0.12 & 0.33 & 62954 & 57.7 & 1.4 & 48.0 \\
\cline { 2 - 8 } & final & 0.12 & 0.29 & 70770 & 58.2 & 1.5 & 48.5 \\
\hline \multirow{2}{*}{ Blue } & initial & 0.06 & 0.28 & 61951 & 57.7 & 1.3 & 47.9 \\
\cline { 2 - 8 } & final & 0.06 & 0.17 & 54721 & 58.0 & 1.4 & 48.1 \\
\hline \multirow{2}{*}{ Red } & initial & 0.20 & 0.39 & 71655 & 58.4 & 1.6 & 47.7 \\
\cline { 2 - 8 } & final & 0.19 & 0.38 & 63288 & 58.2 & 1.5 & 48.5 \\
\hline
\end{tabular}

\section{SINGLE EVENT EFFECTS}

\subsection{Experimental results}

SEE test was performed on 2 samples: \#5 and \#6. The window was removed to allow direct access to the photodiode array of the image sensor by the heavy ions. The properties of the applied beam are shown in the Table 2.

Table 2. Properties of the HI beam and number of events.

\begin{tabular}{|l|l|l|l|l|l|l|l|l|}
\hline Step & Ion & Angle & \multicolumn{1}{|c|}{ LET final } & Fluence & \multicolumn{1}{|c|}{ \#SEU } & \#SEFI & \#SEU & \#SEFI \\
\hline & & $(\mathrm{deg})$ & $\left(\mathrm{MeV} \cdot \mathrm{cm}^{2} / \mathrm{mg}\right)$ & $\left(\mathrm{ions} / \mathrm{cm}^{2}\right)$ & \multicolumn{2}{|c|}{ Sample } & & \\
\hline 1 & $\mathrm{C}$ & 0 & 1.3 & 1.0 & 0 & 0 & 0 & 0 \\
\hline 2 & $\mathrm{Cr}$ & 0 & 16.0 & 1.0 & 0 & 24 & 1 & 19 \\
\hline 3 & $\mathrm{Kr}$ & 0 & 32.4 & 0.1 & 2 & 15 & 1 & 14 \\
\hline 4 & $\mathrm{Kr}$ & 44 & 45.0 & 0.1 & 2 & 19 & 1 & 12 \\
\hline 5 & $\mathrm{Xe}$ & 0 & 62.5 & 0.1 & 4 & 20 & 4 & 24 \\
\hline 6 & $\mathrm{Xe}$ & 39 & 80.4 & 0.1 & 3 & 30 & 4 & 42 \\
\hline 7 & $\mathrm{Xe}$ & 51 & 99.3 & 0.1 & 3 & 25 & 2 & 45 \\
\hline
\end{tabular}

First, two steps were performed with the flux on device of 10,000 ions $/\left(\mathrm{cm}^{2} \cdot \mathrm{s}\right)$ and then 1000 ions $/\left(\mathrm{cm}^{2} \cdot \mathrm{s}\right)$. It was observed that under the higher flux the sensor had some problems with responsivity. All images were taken in a vacuum chamber 
under total darkness and they were acquired continuously with $5 \mathrm{~ms}$ exposure time. All the images were stored for further analysis (SEU events) and all the interruptions due to SEL or SEFI were counted during the test.

The SEL was monitored by means of fast external sources with reaction time in the range of $40 \mu \mathrm{s}$. For these tests it has been considered that a current increase of more than $25 \%$ of the nominal values would present latch-up event. The voltage limits were set to $3.45 \mathrm{~V}$. There was one event in one sensor at the LET level of $62.5 \mathrm{MeV} \cdot \mathrm{cm}^{2} / \mathrm{mg}$ (the $5^{\text {th }}$ step). Since the $\mathrm{LET}_{\text {th }}>60 \mathrm{MeV} \cdot \mathrm{cm}^{2} / \mathrm{mg}$ which is the limit for the mission, no more analysis was performed for SEL. The SEU is usually monitored in the registers, sending a pattern of registers, and monitoring them continuously until a bit flip occurs. Counting an event and repeating the task again until the desired fluence is reached. The registers of the investigated sensor are only accessible in write mode and no readout is possible (only four registers). Therefore, any change/corruption of the image meant that there was change in one of the four registers. Such changes were accounted as an SEU (see Figure 6 with example of corrupted images).

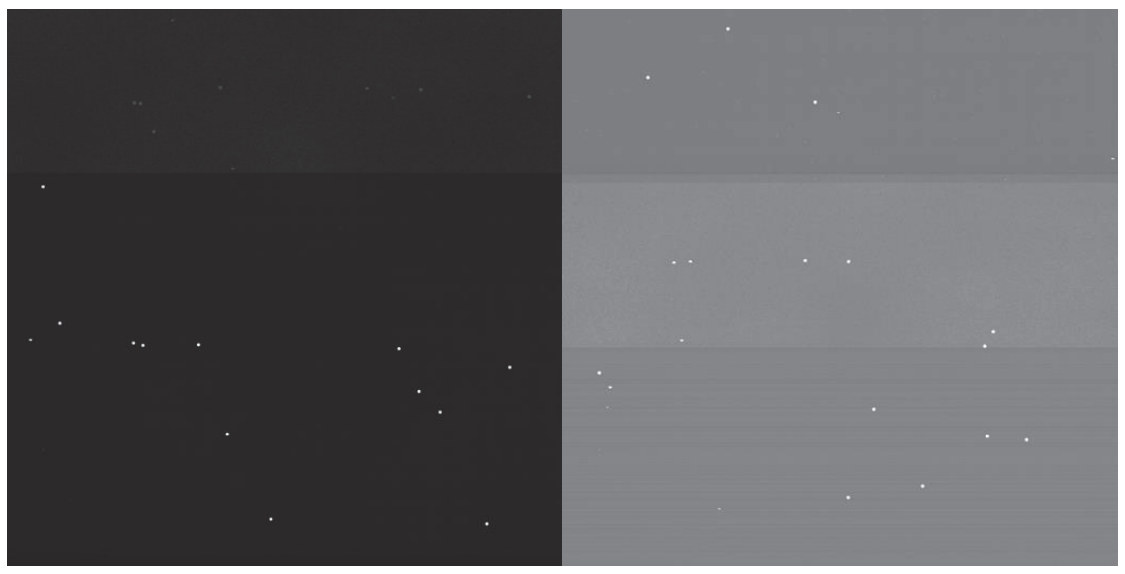

Figure 6. Example of the SEU reflected in the images.

A SEFI is any event which makes image sensor unresponsive, but different from the SEL. It could be caused by SEU in the control register. Considering the comment about the writing mode of the registers, a SEFI was registered when the error caused in the registers (first seen as SEU) was repeated in more than one image, namely the sensor did not recover and was sending corrupted images again. In this case a restart of the camera was necessary. Based on the analysis of the images the numbers of corrupted images and the required restarts for each step are shown in the Table 2. Furthermore, a full characterization was performed before and after the test and the comparison is shown in the Table 3 .

Table 3. Comparison of PTM parameters after SEE test.

\begin{tabular}{|l|l|l|l|l|l|l|l|}
\hline Color & Test & Responsivity & $\begin{array}{c}\text { Quantum } \\
\text { efficiency }\end{array}$ & $\begin{array}{c}\text { Saturation } \\
\text { capacity }\end{array}$ & $\begin{array}{c}\text { Dynamic } \\
\text { range }\end{array}$ & $\begin{array}{c}\text { Linearity } \\
\text { error }\end{array}$ & $\begin{array}{c}\text { SNR } \\
\text { max }\end{array}$ \\
\hline \multirow{3}{*}{ Yellow } & & $(\mathrm{A} / \mathrm{W})$ & & $(\mathrm{e}-)$ & $(\mathrm{dB})$ & $(\%)$ & $(\mathrm{dB})$ \\
\cline { 2 - 8 } & finitial & 0.17 & 0.34 & 66836 & 58.2 & 1.4 & 48.2 \\
\hline \multirow{2}{*}{ Green } & initial & 0.17 & 0.35 & 63457 & 58.8 & 1.5 & 48.0 \\
\cline { 2 - 8 } & final & 0.14 & 0.33 & 77190 & 58.2 & 1.3 & 48.9 \\
\hline \multirow{2}{*}{ Blue } & initial & 0.08 & 0.30 & 65959 & 58.6 & 1.5 & 48.2 \\
\cline { 2 - 8 } & final & 0.07 & 0.21 & 64924 & 57.7 & 1.3 & 48.9 \\
\hline \multirow{2}{*}{ Red } & initial & 0.22 & 0.18 & 60287 & 58.1 & 1.5 & 47.8 \\
\cline { 2 - 8 } & final & 0.21 & 0.43 & 75120 & 58.1 & 1.4 & 48.7 \\
\hline
\end{tabular}


The initial values of the responsivity are even higher than $12 \%$ in respect to the ones for the TID samples. It is assumed that this must be attributed to the window characteristics allowing more photons to reach the surface of the image sensor. After the SEE test all parameters show some degradation irrespective of the pixel color. The most affected parameter is the saturation capacity and the quantum efficiency which decreased within a $14 \%$ margin.

\subsection{Calculation of the SEU and SEFI rates}

To calculate the SEE rates, the models implemented in the CRÈME96 software were used $[12,13,14]$. In principle, there are three main steps in the calculation of the SEE rates: (i) Measure the cross section $\sigma$ using accelerator testing, where the device cross section is defined as the ratio of the number of upsets to the particle fluence. (ii) Determine the sensitive volume (SV) - usually SV is smaller than the actual device physical volume, and it is the most difficult parameter to estimate. One possible way is to use the Weibull fit to estimate the bit size. (iii) Finally, to determine the device error rate, the cross section and sensitive device volume must be integrated with the LET spectrum. The third step can be calculated by means of CRÈME96 including the calculation of the desired LET spectrum. CRÈME96 calculates the SEE rate due to direct ionization using the Rectangular Parallelepiped (RPP) model.

The Weibull function is widely used to fit direct ionization (heavy-ion) SEE cross-section data, since it provides great flexibility in fitting the "turn-on" in the cross-section and naturally levels to a plateau or limiting value. The functional form of Weibull fit reads:

$$
\sigma(x)=\sigma_{0}\left(1-\exp \left[-\left(\frac{x-x_{0}}{W}\right)^{S}\right]\right)
$$

where $x$ is effective LET in $\mathrm{MeV} \cdot \mathrm{cm}^{2} / \mathrm{mg}, \sigma(x)$ is the SEE cross-section in $\mu \mathrm{m}^{2} / \mathrm{bit} ; \sigma_{0}$ is the limiting or plateau crosssection in $\mu \mathrm{m}^{2} /$ bit, $x_{0}$ is the onset parameter (LET threshold) in $\mathrm{MeV} \cdot \mathrm{cm}^{2} / \mathrm{mg}, W$ is the width parameter in $\mathrm{MeV} \cdot \mathrm{cm}^{2} / \mathrm{mg}$, and $S$ is a dimensionless exponent. Using the number of corrupted images and the effective fluence, the cross sections were calculated for each event. After Weibull fit it was found that LET threshold is higher than $15 \mathrm{MeV} \cdot \mathrm{cm}^{2} / \mathrm{mg}$ for both, SEU and SEFI. The plateau cross-section for SEFI is of about $4 \cdot 10^{-6} \mathrm{~cm}^{2} / \mathrm{bit}$, whereas for SEU reads $6 \cdot 10^{-5} \mathrm{~cm}^{2} / \mathrm{bit}$, which is one order of magnitude less than that reported by Beaumel [10] for the same image sensor.

According to the JUICE requirements for the SEE rates estimation following data were used:

- Orbit: geostationary or interplanetary mission (which is the same in the CRÈME96).

- Environment: quiet (solar minimum, which is equivalent to galactic cosmic ray (GCR) maximum), worst week, peak 5 minutes.

- Shielding provided between the device and the external space radiation environment was Al $1 \mathrm{~g} / \mathrm{cm} .^{2}$

- Device size (volume) - the bit is $18 \mu \mathrm{m} \times 18 \mu \mathrm{m} \times 6 \mu \mathrm{m}$.

- SEE cross-sections due to direct ionization presented above.

First, the flux for GEO orbit and ions with Z numbers from 1 to 92 was calculated for the three environments: (i) Solar Minimum (GCR Maximum), (ii) Worst Week, and (iii) Peak 5 Minutes of the Solar Event. Then the transport of the input flux through the thickness of the shielding of Al with $1 \mathrm{~g} / \mathrm{cm}^{2}$ was obtained. Upon finalizing the previous task, the LET spectra for the three environments were calculated. Finally, using the LET spectra, the output of the Weibull fits of the cross section vs. LET, and assuming the depth of the sensitive volume as $6 \mu \mathrm{m}$, the SEU rates were obtained for GEO orbit for 1 bit. To calculate the rates for the entire device it was necessary to multiply 1-bit result by number of bits in the device. The HAS2 image sensor has 2 shift registers ( $Y$ and X) with 10 bits each, one control register with 10 bits, and 12-bit ADC resulting in 42 bits which can be flipped causing SEU. In principle, for the SEFI rates, only the control register plays significant role, but taking the worst case, also 42 bits were considered. The calculated rates for three environments are shown in the Table 4.

Obtained rates confirm the resistance of this imager to the SEE. Even in the worst conditions, namely peak 5 minutes, the probability of the SEU is less than one event per day. It is necessary to note that in space the flux of high energetic particles (i.e. heavy ions) is of about 4 particles $/\left(\mathrm{cm}^{2} \cdot \mathrm{s}\right)$ and all the tests in the labs need to be considered as accelerated tests. The obtained rates are very small, leading to the probability of less than $1 \%$ that the SEFI event occurs during the 10 -year mission. 
Table 4. SEU and SEFI rates calculated by means of CREME96.

\begin{tabular}{|c|c|c|}
\hline Environment & SEU & SEFI \\
\hline & events/device/day & events/device/day \\
\hline Quiet & $1.4 \mathrm{e}-04$ & $1.6 \mathrm{e}-05$ \\
\hline Worst week & $1.2 \mathrm{e}-02$ & $1.5 \mathrm{e}-03$ \\
\hline Peak 5 minutes & $1.3 \mathrm{e}-01$ & $1.5 \mathrm{e}-02$ \\
\hline
\end{tabular}

\section{CONCLUSIONS}

The HAS2 image sensor with custom CFA was tested under different radiation conditions: TID and SEE. Results obtained during the TID test confirm that both, sensor and CFA, are radiation hardened against gamma radiation of up to $57 \mathrm{krad}$. As expected, the most affected parameter was the dark current which increased $40 \%$ during the radiation test but then partially recovered after annealing at higher temperature. The SEE test revealed that the imagers are SEL free up to $62.5 \mathrm{MeV} \cdot \mathrm{cm}^{2} / \mathrm{mg}$. Moreover, obtained rates for SEU and SEFI in the range of $10^{-4}$ and $10^{-5}$ events/device/day led to probabilities of less than $1 \%$ that these events occur during the planned 10-year of ESA's JUICE mission. The rates increase substantially in the worst week ( 2 orders of magnitude) and peak 5 minutes ( 3 orders of magnitude) but still are very low, less than one event per day. The peak 5 minutes environment refers to flares which occur seldom, and there are three known cases from the beginning of the monitoring of such events. It is necessary to bear in mind that the window on the image sensor and the camera optics will be an additional barrier, blocking the incoming heavy ions, thus reducing the SEE rates. At the other hand, the heavy ions still can be produced inside the sensor cavity due to nuclear reactions caused by the high energy protons $(>70 \mathrm{MeV})$, however this process has lower probability. The results of the here described evaluation confirm that the selection of the HAS2 sensors with the CFA proposed by Micro-Cameras and Space Exploration SA and for its JUICE Monitoring Cameras was a valuable decision and that it fits well the mission profile.

\section{REFERENCES}

[1] JUICE Team, "JUICE Environment Specification," JS-14-09(5.5), 1-133 (2017).

[2] Wang, M., Ahn, J.H., Jiang, L., Shi, W., Son, S., Park, Y.J., and Ryu, J.H., " Ocean color products from the Korean Geostationary Ocean Color Imager (GOCI)," Opt. Express 21, 3835-3849 (2013).

[3] Martin-Gonthier, P., Magnan, P., Corbiere, F., Rolando, S., Saint-Pe, O., Breart de Boisanger, M., and Larnaudie, F., "CMOS Detectors for Space Applications: From R\&D to operational program with large volume foundry," Proc. SPIE 7826, 78261P (2010).

[4] Korendyke, C.M., Vourlidas, A., Plunkett, S.P., Howard, R.A., Wang, D., Marshall, C.J., Waczynski, A., Janesick, J.J., Elliott, T., Tun, S., Tower, J., Grygon, M., Keller, D., and Clifford, G.E., "Development and test of an active pixel sensor detector for heliospheric imager on solar orbiter and solar probe plus," Proc. SPIE 8862, $88620 \mathrm{~J}(2013)$.

[5] Della Corte, V., Schmitz, N., Zusi, M., Castro, J.M., Leese, M., Debei, S., Magrin, D., Michalik, H., Palumbo, P., Jaumann, R., Cremonese, G., Hoffmann, H., Holland, A., Lara, L.M., Fiethe, B., Friso, E., Greggio, D., Herranz, M., Koncz, A., Lichopoj, A., Martinez-Navajas, I., Mazzotta Epifani, E., Michaelis, H., Ragazzoni, R., Roatsch, T., Rodrigo, J., Rodriguez, E., Schipani, P., Soman, M., Zaccariotto, M., and the International JANUS team, "The JANUS camera onboard JUICE mission for Jupiter system optical imaging," Proc. SPIE 9143, 91433I (2014).

[6] De Groof, A., Berghmans, D., Nicula, B., Halain, J.P., Defise, J.M., Thibert, T., and Schüle, U., "CMOS-APS Detectors for Solar Physics: Lessons Learned during the SWAP Preflight Calibration," Solar Phys. 249, 147-163 (2008).

[7] Beaumel, M., Hervé, D., and Van Aken, D., "Cobalt-60, Proton and Electron Irradiation of a Radiation-Hardened Active Pixel Sensor," IEEE Trans. Nucl. Sci. 57(4), 2056-2065 (2010).

[8] Inguimbert, C., Nuns, T., Ursule, M. C., Falguère, D., Hervé, D., Beaumel, M., and Poizat M., "Modeling the Dark Current Non-Uniformity of Image Sensors with GEANT4," IEEE Trans. Nucl. Sci. 61(6), 3323-3330 (2014) 
[9] Van Aken, D., Hervé, D., and Beaumel, M., "Total Dose, Displacement Damage and Single Event Effects in the Radiation Hardened CMOS APS HAS2," Proc. SPIE 7474, 74741C (2009).

[10] Beaumel, M., Hervé, D., Van Aken, D., Pourrouquet, P., and Poizat, M., "Proton, Electron, and Heavy Ion Single Event Effects on the HAS2 CMOS Image Sensor," IEEE Trans. Nucl. Sci. 61(4), 1909-1917 (2014)

[11] Xiao, H., Hajdas, W., Beauvivre, S., Kraehenbuehl, D., Ziethe, R., and Banerji, N., "Simulations of radiation damage in spacecraft camera for ESA JUICE mission," Proc. RADECS 2018, 1-4 (2018)

[12] Tylka, A.J., Adams Jr., J.H., Boberg, P.R., Brownstein, B., Dietrich, W.F., Flueckiger, E.O., Petersen, E.L., Shea, M.A., Smart, D.F., and Smith, E.C., "CREME96: A Revision of the Cosmic Ray Effects on Microelectronics Code, " IEEE Trans. Nucl. Sci. 44(6), 2150-2160 (1997).

[13] Weller, R.A., Mendenhall, M.H., Reed, R.A., Schrimpf, R.D., Warren, K.M., Sierawski, B.D., and Massengill, L.W., "Monte Carlo simulation of single event effects," IEEE Trans. Nucl. Sci. 57(4) 1726-1746 (2010).

[14] Mendenhall M.H., and Weller, R.A., "A probability-conserving cross-section biasing mechanism for variance reduction in Monte Carlo particle transport calculations," Nucl. Inst. \& Meth. A 667, 38-43 (2012). 